\title{
SPIRAL ARMS IN THE DISK OF HD 142527 FROM CO EMISSION LINES WITH ALMA
}

\author{
V. Christiaens ${ }^{1}$, S. Casassus ${ }^{1}$, S. Perez ${ }^{1}$, G. van der Plas ${ }^{1}$, and F. Ménard ${ }^{2}$ \\ ${ }^{1}$ Departamento de Astronomía, Universidad de Chile, Casilla 36-D, Santiago, Chile; valchris@ das.uchile.cl \\ 2 UMI-FCA, CNRS/INSU, France (UMI 3386) and Departamento de Astronomía, Universidad de Chile, Santiago, Chile \\ Received 2014 January 24; accepted 2014 March 5; published 2014 March 28
}

\begin{abstract}
In view of both the size of its gap and the previously reported asymmetries and near-infrared spiral arms, the transition disk of the Herbig Fe star HD 142527 constitutes a remarkable case study. This paper focuses on the morphology of the outer disk through ALMA observations of ${ }^{12} \mathrm{CO} J=2-1,{ }^{12} \mathrm{CO} J=3-2$, and ${ }^{13} \mathrm{CO} J=2-1$. Both ${ }^{12} \mathrm{CO} J=2-1$ and ${ }^{12} \mathrm{CO} J=3-2$ show spiral features of different sizes. The innermost spiral arm (S1) is a radio counterpart of the first near-infrared spiral observed by Fukagawa, but it is shifted radially outward. However, the most conspicuous CO spiral arm (S2) lies at the outskirts of the disk and has not been detected before. It corresponds to a cold density structure, with both brightness and excitation temperatures of order $13 \pm 2 \mathrm{~K}$ and conspicuous in the ${ }^{12} \mathrm{CO} J=2-1$ peak-intensity map, but faint in ${ }^{12} \mathrm{CO} J=3-2$. There is also a faint counterarm (S3), at a point-symmetric location of S2 with respect to the star. These three spirals are modeled separately with two different formulae that approximate the loci of density maxima in acoustic waves due to embedded planets. S1 could be fit relatively well with these formulae, compared to S2 and S3. Alternative scenarios such as gravitational instability or external tidal interaction are discussed. The impact of channelization on spectrally and spatially resolved peak intensity maps is also briefly addressed.
\end{abstract}

Key words: planet-disk interactions - protoplanetary disks - stars: individual (HD 142527)

Online-only material: color figures

\section{INTRODUCTION}

The young circumstellar disks that host a large annular gap or central cavity, the so-called transition disks (TDs), could be crucial in the context of planetary formation as their morphologies may result from dynamical clearing due to planet(s) (Dodson-Robinson \& Salyk 2011; Zhu et al. 2012, 2013), rather than dust grain, growth, or photo-evaporation alone (Rosotti et al. 2013).

Asymmetric features, warps, or spirals in TDs can also be evidence of the presence of stellar or planetary companions (e.g., Goldreich \& Tremaine 1979). Spiral features have mostly been detected in optical or near-infrared (NIR) wavelengths (e.g., Grady et al. 2001, 2013; Clampin et al. 2003; Fukagawa et al. 2004; Muto et al. 2012), and in the disk of AB Aur at radio wavelengths (Corder et al. 2005; Lin et al. 2006), although in counter-rotation with the disk, thus probably stemming from a late envelope infall (Tang et al. 2012).

The disk around the Herbig Fe star HD 142527 constitutes an outstanding example of a nearly face-on TD $\left(i \sim 28^{\circ} \pm 3^{\circ}\right.$; Perez et al. 2014), showing both a large asymmetric dust depleted gap and a spiral arm observed in NIR scattered light (Fukagawa et al. 2006). Its mass and age were estimated to be $\sim 1.9-2.2 M_{\odot}$ and 1-12 Myr old, respectively (Fukagawa et al. 2006; Verhoeff et al. 2011), for a distance of $145 \pm 15 \mathrm{pc}$ (de Zeeuw et al. 1999; Acke \& van den Ancker 2004). Fujiwara et al. (2006) concluded that the disk's rotation axis was inclined so that the east side was the far side, with a position angle (P.A.) of $\sim-20^{\circ}$. With $K$-band imaging, Casassus et al. (2012) reported the presence of smaller spiral structures at the outer edge of the gap, confirmed by Rameau et al. (2012) and in NIR polarized intensity studies (Canovas et al. 2013; Avenhaus et al. 2014, the latter work identifies two new spirals).

ALMA provided substantial insight into the structure of HD 142527. Casassus et al. (2013b) found gap-crossing $\mathrm{HCO}^{+}(4-3)$ filaments, diffuse $\mathrm{CO}(3-2)$ inside the cavity, and confirmed the horseshoe morphology of the dust continuum reported by Ohashi (2008), which they interpreted as a dust trap. Fukagawa et al. (2013) reported on another ALMA data set in ${ }^{13} \mathrm{CO} J=3-2, \mathrm{C}^{18} \mathrm{O} J=3-2$, and underlying continua.

Here, we focus on the outskirts of the HD 142527 disk and report on the discovery of several CO spiral arms.

\section{OBSERVATIONS}

\subsection{Data Sets}

The observations were obtained with ALMA on 2012 June (Cycle 0) and cover ${ }^{12} \mathrm{CO} J=2-1(230.538 \mathrm{GHz}$, hereafter CO $2-1),{ }^{12} \mathrm{CO} J=3-2(345.796 \mathrm{GHz}, \mathrm{CO} 3-2)$, and ${ }^{13} \mathrm{CO}$ $J=2-1\left(220.399 \mathrm{GHz},{ }^{13} \mathrm{CO} 2-1\right)$, with baselines comprised between 21 (resolution of $\sim 12^{\prime \prime} .8$ ) and $379 \mathrm{~m}\left(\sim 0^{\prime \prime} .71\right)$. For details on the instrumental setup and calibration, we refer to Perez et al. (2014) and Casassus et al. (2013b), respectively, for bands 6 (CO 2-1 and $\left.{ }^{13} \mathrm{CO} 2-1\right)$ and 7 (CO 3-2).

Spectral line imaging was achieved with the CASA package. The continuum was first subtracted in the visibility domain. We binned the original spectral channels to $0.2 \mathrm{~km} \mathrm{~s}^{-1}$ bin-width. Next, visibility data were cleaned (Cotton-Schwab CLEAN). As faint extended structures were sought, natural weighting was preferred over Briggs weighting, and masks were drawn manually in the dirty maps for each velocity channel. To avoid spurious detections, the masks delimited signal at $\sim 5 \sigma$. For comparison, an elliptical mask identical in all velocity channels, embracing the whole butterfly pattern, yielded similar moment maps.

\subsection{Moment Maps}

Inspection of the channel maps for the different $\mathrm{CO}$ transitions confirms the LSR systemic radial velocity of $3.7 \pm 0.2 \mathrm{~km} \mathrm{~s}^{-1}$ (Casassus et al. 2013b; Perez et al. 2014). Both $\mathrm{CO} 2-1$ and $\mathrm{CO} 3-2$ (but not ${ }^{13} \mathrm{CO} 2-1$ ) channels with 


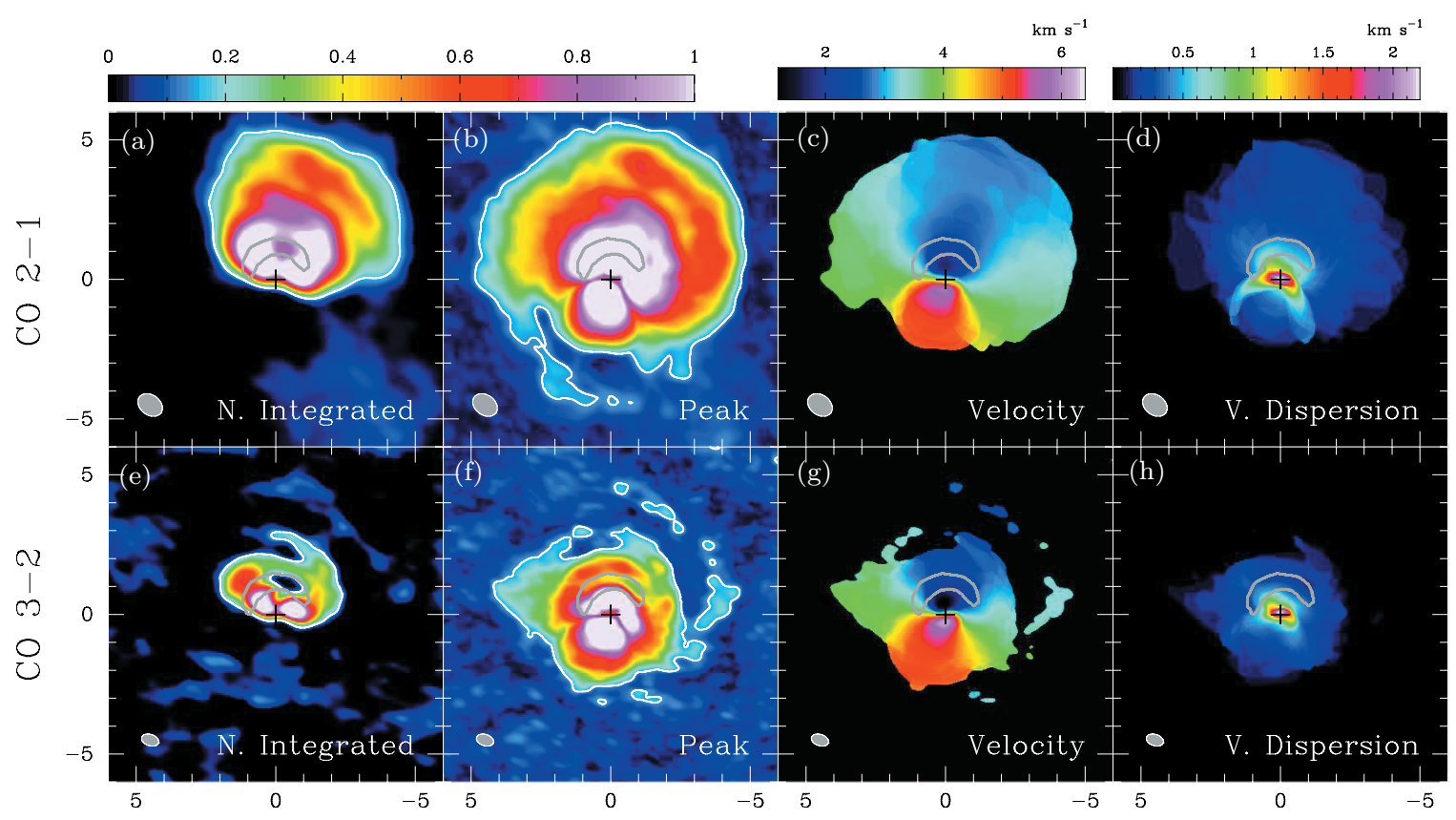

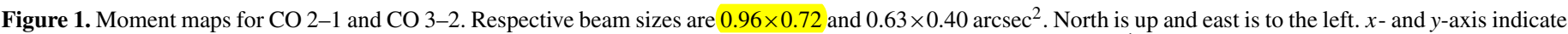

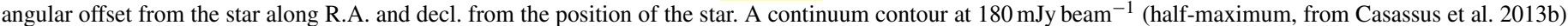

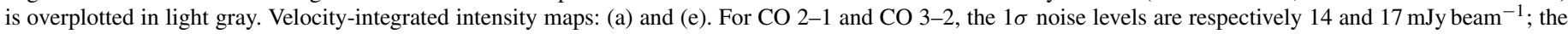

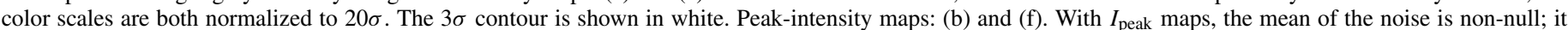

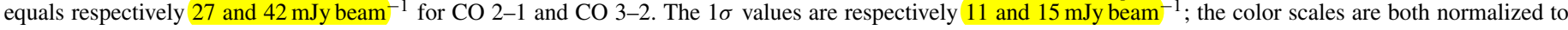
$40 \sigma$. The relevant contour, mean(noise) $+3 \sigma$, is shown in white. Velocity centroid: (c) and (g). Velocity dispersion: (d) and (h).

(A color version of this figure is available in the online journal.)

velocity higher than the systemic velocity show a decrement in the specific intensity levels between 4.2 and $4.8 \mathrm{~km} \mathrm{~s}^{-1}$ (southern velocities). These velocities correspond to an extended diffuse cloud best detected in single-dish data (Casassus et al. 2013a).

For $\mathrm{CO} 2-1$ and $\mathrm{CO} 3-2$ lines, maps of the velocityintegrated intensity $\left(I_{\text {int }}\right)$, the peak intensity $\left(I_{\text {peak }}\right)$, and the velocity centroid and dispersion are computed in order to further characterize the outer disk (Figure 1). The intensity scale is chosen to emphasize the fainter structure in the outer disk, at the expense of saturation in the brighter central regions, discussed in detail by Casassus et al. (2013b) and Perez et al. (2014). We restrict the velocity range to $2.6<v_{\mathrm{lsr}} / \mathrm{km} \mathrm{s}^{-1}<3.6$ (northern velocities) for the computation of the $I_{\text {int }}$ maps because of the intervening cloud.

The computation of the $I_{\text {peak }}$ maps revealed an unknown issue. As seen in Figure 2(a), the signal in the disk appears segmented for $\mathrm{CO} 2-1$ (this is also the case for $\mathrm{CO} 3-2$ ). This might be due to the discretization of the velocity field in channelaveraged visibilities. To prove that the segmentation is spurious, we computed two (four) $I_{\text {peak }}$ maps with the same velocity bin width of $0.2 \mathrm{~km} \mathrm{~s}^{-1}$, but shifted by half (a quarter of) the bin width, and then averaged them. The fragmentation follows the velocity channels and is almost completely smoothed out when averaging the different binnings (Figures 2(b) and (c)).

From the CO 2-1 $I_{\text {int }}$ map in the north (Figure 1(a)), an arc-like structure in the NW of the image, already hinted in the channel maps, is detected at very large distance from the star ( 3".6). Closer in, at about 2".4, the intensities are higher and more extended to the NNW than to the NNE. In the $I_{\text {peak }}$ map (Figure 1(b)), the closer-in structure turns out to be shaped roughly as a spiral arm (hereafter $\mathrm{S} 1$ ), whereas the large-scale arc is revealed as a conspicuous tightly wound spiral (S2). At an approximately point-symmetric location of $\mathrm{S} 2$ with respect to the star, we also detect a remarkable counterspiral (S3).
We estimated an upper limit on the flux loss due to interferometer filtering by aperture photometry in the relevant channels of a LIME (Brinch \& Hogerheijde 2010) model of HD 142527 (Perez et al. 2014). Cleaning the model, sampled at the uvcoverage of our observation, and comparing with the original model yields flux losses $\lesssim 16 \%$. While this result suggests that filtering out is minor for $\mathrm{S} 2$, the faintness of $\mathrm{S} 3$ probably stems from absorption due to the intervening cloud.

Bridge-like features in the central regions of the $I_{\text {peak }}$ maps (Figures 1(b) and (f)), also seen in the velocity dispersion map (Figures 1(d) and (h)), are due to this intervening cloud. The kinematics in CO 2-1 appear to be Keplerian, even under spirals $\mathrm{S} 1$ and S2. The spirals do not have counterparts in velocity dispersion, i.e., there is no local enhancement of the velocity gradient or turbulence.

The CO 3-2 $I_{\text {int }}$ map in the north (Figure 1(e)) displays S1 more evidently, and the $I_{\text {peak }}$ map (Figure 1(f)) also confirms its presence. The faint patches of signal just above the mean (noise) $+3 \sigma$ level to the NW in Figure 1(f) follow the shape and location of S2, as first detected in Figure 1(b).

However, the outer disk seems too faint to enable detection of extended structures in ${ }^{13} \mathrm{CO} 2-1$.

\subsection{Spiral Arms}

The three spiral arms extend in projected angular separations, but deprojected physical distances:

1. from $\sim 1^{\prime \prime} .9(\sim 290 \mathrm{AU})$ at P.A. $\sim-110^{\circ}$ to $\sim 2^{\prime \prime} .8(\sim 380 \mathrm{AU})$ at P.A. $\sim 0^{\circ}$ for $\mathrm{S} 1$;

2. from $\sim 3^{\prime \prime} .0(\sim 520 \mathrm{AU})$ at P.A. $\sim-100^{\circ}$ to $\sim 4^{\prime \prime} .2(\sim 640 \mathrm{AU})$ at P.A. $\sim 0^{\circ}$ for $\mathrm{S} 2$;

3. from $\sim 3^{\prime \prime} .2(\sim 520 \mathrm{AU})$ at P.A. $\sim 100^{\circ}$ to $\sim 4^{\prime \prime} .4(\sim 670 \mathrm{AU})$ at P.A. $\sim 190^{\circ}$ for $\mathrm{S} 3$. 


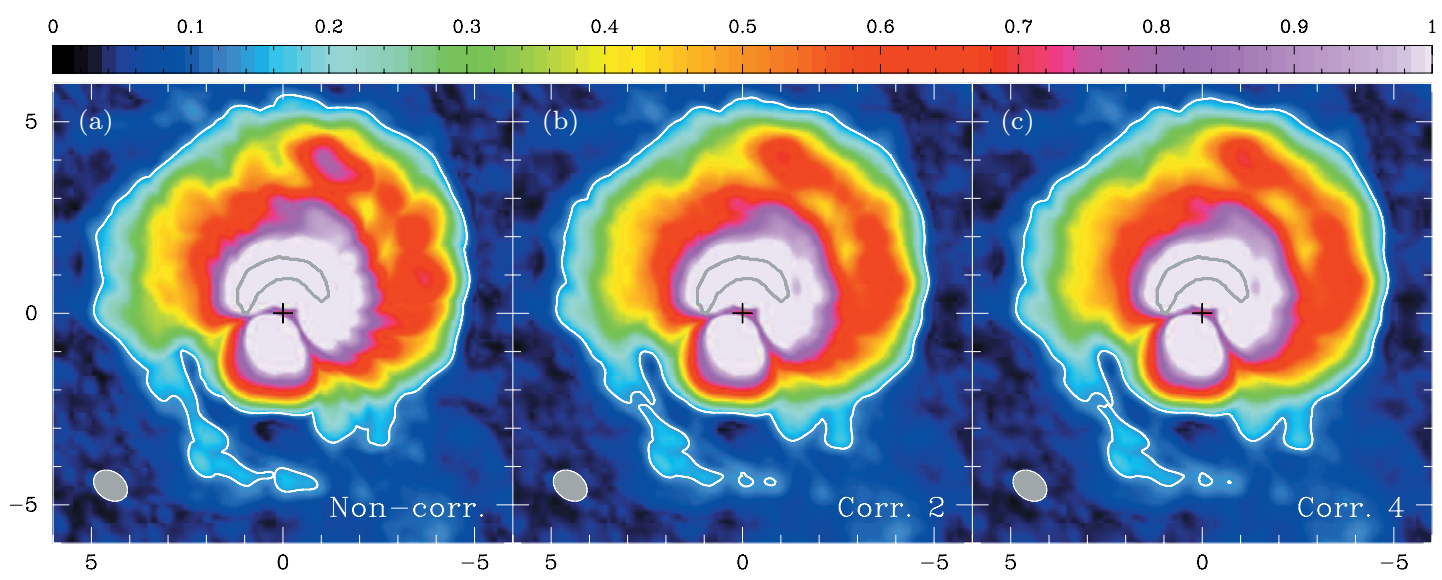

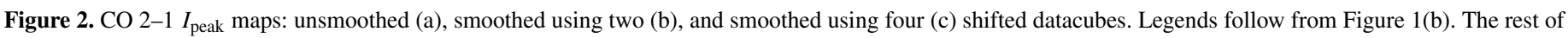
the paper implicitly shows $I_{\text {peak }}$ maps smoothed using four shifted datacubes.

(A color version of this figure is available in the online journal.)

They all subtend $\sim 100^{\circ}$ in azimuth. S2 and S3 have radial widths ranging $00^{\prime \prime} 6-1{ }^{\prime \prime} 1(90-160 \mathrm{AU})$ and are thus globally resolved radially.

\subsubsection{Physical Diagnostics in the Spiral Arms}

Except for S3, whose signal is absorbed, several physical parameters describing the spirals may be derived. In $I_{\text {peak }}(\mathrm{CO}$ $2-1$ ), the maximum intensities are $\sim 450 \mathrm{mJy} \mathrm{beam}^{-1}$ for S1 (at P.A. $\sim-70^{\circ}, R \sim 330 \mathrm{AU}$ ) and $\sim 280 \mathrm{mJy}^{\circ}$ beam $^{-1}$ for $\mathrm{S} 2$ (at P.A. $\left.\sim-80^{\circ}, R \sim 530 \mathrm{AU}\right)$. In $I_{\text {peak }}(\mathrm{CO} 3-2)$, the maximum intensities, roughly at the same locations as for $\mathrm{CO} 2-1$, are

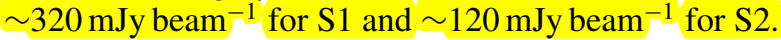

Assuming that ${ }^{12} \mathrm{CO}$ is optically thick, the peak intensities provide brightness temperatures, which approximate the kinetic temperatures at the unit-opacity surfaces, of $T_{b, S 1} \sim 20 \mathrm{~K}$ (from both $\mathrm{CO} 2-1$ and $\mathrm{CO} 3-2$ maximum intensities) and $T_{b, S 2} \sim 11-15 \mathrm{~K}$ (the upper value stems from $\mathrm{CO} 2-1$ maximum intensity). Assuming LTE, the excitation temperatures derived from the ratio of $\mathrm{CO} 2-1$ and $\mathrm{CO} 3-2$ maximum intensities are $T_{\mathrm{ex}, S 1} \sim 22-27 \mathrm{~K}$ and $T_{\mathrm{ex}, S 2} \sim 13-15 \mathrm{~K}$.

CO gas is expected to condense on dust for $T \lesssim 19-20 \mathrm{~K}$ (e.g., Qi et al. 2011; de Gregorio-Monsalvo et al. 2013). The observation of $\mathrm{CO}$ gas at lower temperature in $\mathrm{S} 2$ may suggest that dust grains are depleted, or mainly settled in the mid-plane (e.g., Dullemond \& Dominik 2004), or that CO desorption is efficient at the surface (Hersant et al. 2009) at that radial distance.

The gas temperature in the gap is $\sim 41-43 \mathrm{~K}$ (Fukagawa et al. 2013; Perez et al. 2014). Using this value at $80 \mathrm{AU}, 20-27 \mathrm{~K}$ at $330 \mathrm{AU}\left(T_{b, S 1}-T_{\mathrm{ex}, S 1}\right)$, and $13-15 \mathrm{~K}$ at $530 \mathrm{AU}\left(T_{b, S 2}-T_{\mathrm{ex}, S 2}\right)$, the best fit to the law $T \propto r^{-q}$ yields $q \sim 0.5-0.6$. This range of values is steeper than $q \sim 0.3$ found by Perez et al. (2014) up to $\sim 300 \mathrm{AU}$.

Sound speed and scale height are defined respectively as $c_{s}^{2}=k_{B} T_{b} /\left(\mu m_{p}\right)$ and $H=c_{s} / \Omega$, where $k_{B}$ is the Boltzmann constant; $\mu$ is the mean molecular weight of the gas in terms of proton mass $m_{p}$, namely $\sim 2.3$ for $\mathrm{H}_{2}$ with $10 \%$ of He; and $\Omega$ is the angular frequency at the physical radius where the temperature is derived. With $T_{S 1} \sim 20-27 \mathrm{~K}$ and $T_{S 2} \sim 13-15 \mathrm{~K}$, and assuming Keplerian rotation, we find scale heights of $H_{S 1} \sim 38-44 \mathrm{AU}$ and $H_{S 2} \sim 66-76 \mathrm{AU}$, and hence a disk aspect ratio $h_{S} \sim 0.12-0.13$ at the location of both spirals. Verhoeff et al. (2011) quoted a scale height of $60 \mathrm{AU}$ at the inner rim of the outer disk $(h \sim 0.46)$. However, considering a disk with $i \sim$ $28^{\circ} \pm 3^{\circ}$ (Perez et al. 2014) instead of $20^{\circ}$ leads to a scale height of $20 \pm 7 \mathrm{AU}(h \sim 0.15 \pm 0.05)$ in the inclination/inner rim scale height relation of Avenhaus et al. (2014), thus in agreement with our result.

We can also obtain lower limits on the mass of each spiral in LTE. The integrated flux was computed in S1 and S2 from $I_{\text {peak }}(\mathrm{CO} 2-1)$ by defining an aperture $1^{\prime \prime}$ wide in radius and centered on the best fit model spiral to the Kim (2011) equation (see Section 2.3.2). We obtain integrated fluxes of $1.41 \mathrm{Jy}$ and $1.27 \mathrm{Jy}$ for $\mathrm{S} 1$ and $\mathrm{S} 2$, respectively. Using these values with $T_{\text {ex }, S 1}$ and $T_{\text {ex }, S 2}$, a velocity dispersion of $\sim 0.3 \mathrm{~km} \mathrm{~s}^{-1}$ (see Figure $1(\mathrm{~d})$ ), and assuming $\left[\mathrm{H}_{2}\right] /[\mathrm{CO}]=10^{4}$, the total gas mass in each spiral is $M_{S 1} \gtrsim 1.38 \times 10^{-6} M_{\odot}$ and $M_{S 2} \gtrsim$ $1.23 \times 10^{-6} M_{\odot}$.

\subsubsection{Modeling of the Spiral Arms}

An elliptical grid is defined to match the inclination and P.A. of the projected disk, with deprojected bins of 0 '. 125 in radius and $5^{\circ}$ in azimuth. The trace of each spiral is identified with radial cuts in $I_{\text {peak }}$, using local maxima for S2 and S3, and local inflection points for S1 (second-derivative nulls). We obtain 16,20 , and 20 points tracing, respectively, S1, S2, and S3 in the CO 2-1 $I_{\text {peak }}$ map (Figure 3(a)), and 17 and 14 points, respectively, tracing $\mathrm{S} 1$ and $\mathrm{S} 2$ in the $\mathrm{CO} 3-2 I_{\text {peak }}$ map (Figure 3(c)). Uncertainties in the measured positions of the spirals were set to $\max \{$ bin size, quarter of the beam size $\}$. We then perform least-squares fits to each spiral independently using two different parametric formulae, as follows.

The polar coordinates $(\theta, r)$ of an acoustic wave created by a planet, at location $\left(\theta_{c}, r_{c}\right)$, on a gaseous disk can be approximated by (Muto et al. 2012 and references therein)

$$
\begin{aligned}
\theta(r) & =\theta_{c}+\frac{\operatorname{sgn}\left(r-r_{c}\right)}{h_{c}} \\
& \times\left\{\left(\frac{r}{r_{c}}\right)^{1+\beta}\left[\frac{1}{1+\beta}-\frac{1}{1-\alpha+\beta}\left(\frac{r}{r_{c}}\right)^{-\alpha}\right]\right. \\
& \left.-\left(\frac{1}{1+\beta}-\frac{1}{1-\alpha+\beta}\right)\right\},
\end{aligned}
$$

where $h_{c}$ is the disk aspect ratio at radius $r_{c}$. It is assumed that $\Omega$ and $c_{s}$ can be expressed as power laws: $\Omega \propto r^{-\alpha}$ and $c_{s} \propto r^{-\beta} \propto r^{-q / 2}$. In total, there are five parameters to each fit $\left(r_{c}, \theta_{c}, h_{c}, \alpha\right.$ and $\left.\beta\right)$. Note that a plus sign was used in 


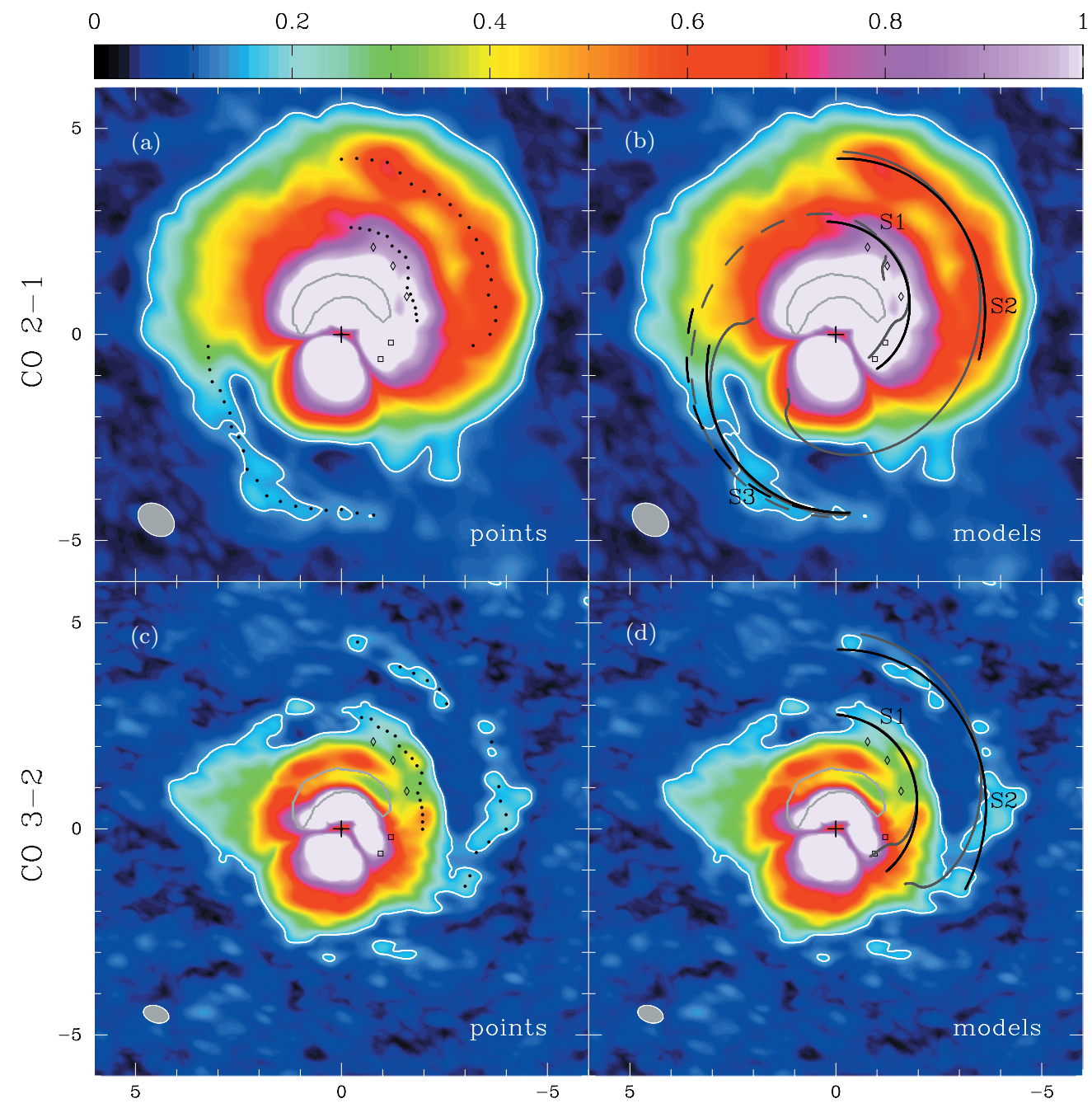

Figure 3. Modeling of the spirals observed in the CO 2-1 and CO 3-2 $I_{\text {peak }}$ maps. Legends follow from Figures 1(b) and (f). For comparison, we indicate the position of the $\mathrm{H}$-band spiral arm from Fukagawa et al. (2006; diamonds) and the Ks-band spiral root from Casassus et al. (2012; squares). (a) Points tracing the spirals used for modeling. (b) Modeling of the spiral arms as in Muto et al. (2012; solid dark gray lines) and Kim (2011; solid black lines). The dashed dark gray and dashed black spirals represent the point-symmetric location of S2 models with respect to the star. (c) and (d) Identical to (a) and (b) with the CO 3-2 $I_{\text {peak }}$ map.

(A color version of this figure is available in the online journal.)

Equation (1) after $\theta_{c}$ instead of the minus sign given in Muto et al. (2012) since the disk of HD 142527 is rotating clockwise.

First fits with Equation (1) were computed by fixing the values of $\alpha$ to 1.5 (Keplerian rotation) and $\beta$ to 0.25 (see $q$ in Section 2.3.1). We found plausible values of $h_{c}$ (between 0.01 and 1.0) with the points of the CO 2-1 $I_{\text {peak }}$ map for S1 $\left(h_{c}=0.15\right)$ and S3 $\left(h_{c}=0.27\right)$, but not for S2, and for neither spiral with the points of the CO 3-2 $I_{\text {peak }}$ map. In order to further reduce the parameter space, a second set of fits was run fixing as well the value of $h_{c}$ to 0.15 . The best fit models are shown in solid dark gray lines in Figures 3(b) and (d); the inflection point in the spiral curves represents the best fit location of the planet. The values of the best fit $r_{c}, \theta_{c}$, and $\chi^{2}$ are given in Table 1 .

Sweeping $\beta$ from 0.15 (Perez et al. 2014) to 0.30 (corresponding to $q=0.6$ ) does not significantly change $\chi^{2}$ (relative to the spread of values for different spirals) and the value of the other parameters. On the contrary, the best fit parameters depend strongly on $h_{c}$; a difference of a few hundredths induces significantly different results, confirming the degeneracy already noted by Muto et al. (2012) and Boccaletti et al. (2013). Since $\chi^{2}$ depends on the estimated error bars, which are not independently determined, we use $\chi^{2}$ to compare different models
Table 1

Values of the Best Fit Parameters with Equations (1) and (2)

\begin{tabular}{|c|c|c|c|c|}
\hline \multicolumn{2}{|c|}{ Equation $(1)^{\mathrm{a}}$} & $\mathrm{S} 1$ & $\mathrm{~S} 2$ & S3 \\
\hline \multirow{3}{*}{ CO 2-1 } & $r_{c}(\operatorname{arcsec})$ & $1.71 \pm 0.04$ & $2.13 \pm 0.24$ & $2.62 \pm 0.08$ \\
\hline & $\theta_{c}(\mathrm{deg})$ & $283 \pm 5$ & $146 \pm 64$ & $83 \pm 10$ \\
\hline & $\chi^{2}$ & 2.38 & 18.0 & 4.66 \\
\hline \multirow{3}{*}{$\mathrm{CO} 3-2$} & $r_{c}(\operatorname{arcsec})$ & $1.62 \pm 0.04$ & $2.69 \pm 0.38$ & l \\
\hline & $\theta_{c}(\operatorname{deg})$ & $255 \pm 7$ & $235 \pm 45$ & l \\
\hline & $\chi^{2}$ & 2.05 & 36.0 & 1 \\
\hline \multicolumn{2}{|c|}{ Equation (2) } & $\mathrm{S} 1$ & $\mathrm{~S} 2$ & S3 \\
\hline \multirow{3}{*}{ CO 2-1 } & $a\left(\operatorname{arcsec} \operatorname{rad}^{-1}\right)$ & $0.58 \pm 0.05$ & $0.20 \pm 0.05$ & $0.65 \pm 0.06$ \\
\hline & $b(\operatorname{arcsec})$ & $-0.86 \pm 0.29$ & $3.05 \pm 0.29$ & $2.34 \pm 0.16$ \\
\hline & $\chi^{2}$ & 0.16 & 0.30 & 0.40 \\
\hline \multirow{3}{*}{$\mathrm{CO} 3-2$} & $a\left(\operatorname{arcsec} \operatorname{rad}^{-1}\right)$ & $0.45 \pm 0.03$ & $0.25 \pm 0.10$ & I \\
\hline & $b(\operatorname{arcsec})$ & $-0.02 \pm 0.17$ & $2.88 \pm 0.52$ & l \\
\hline & $\chi^{2}$ & 0.18 & 2.94 & I \\
\hline
\end{tabular}

Note. ${ }^{\text {a }}$ For Equation (1), we fixed $\alpha=1.5$ (Keplerian rotation), $\beta=0.25$ (from $q$ found in Section 2.3.1), and $h_{c}=0.15$ (best fit value when $h_{c}$ is set free for $\mathrm{S} 1$ in the CO 2-1 $I_{\text {peak }}$ map). 
rather than assess their statistical significance. The best fit spiral appears to be $\mathrm{S} 1$, while $\mathrm{S} 2$ and $\mathrm{S} 3$ provide $\chi^{2}$ values a factor of several times larger.

Another formula suggested by the theoretical work of Kim (2011) approximates the shape of the spiral wake created by an embedded planet on a circular orbit to an Archimedean spiral

$$
r(\theta)=a \theta+b
$$

where $a$ corresponds to $r_{p} / M_{p}$, with $r_{p}$ the launching radius of the planet and $M_{p}$ its Mach number, and $b$ is a constant. Parameters for the best fit of each spiral are given in Table 1. With a $\chi^{2}$ value at least twice as small as the two other spirals, it is again $S 1$ that is the best fit. Equations (1) and (2) are fundamentally different- $\theta(r)$ versus $r(\theta)$-so that $\chi^{2}$ obtained with each equation should not be mutually compared.

The similar slopes $a$ of S3 and S1 may suggest that S3 is elongating S1. Conversely, models for S2 and S3 do not share the same slope, even though $\mathrm{S} 1$ and $\mathrm{S} 2$ roughly coincide with the point-symmetric location of S2 with respect to the star (dashed dark gray curve in Figure 3(b)).

\subsubsection{Comparison with NIR Spiral Arms}

Although S2 and S3 have not been detected before, a spiral arm similar to $\mathrm{S} 1$ has already been reported in the NIR $H$ and $K$ bands by Fukagawa et al. (2006). Also in NIR, closer-in observations in the $K s$-band (Casassus et al. 2012), $L^{\prime}$-band (Rameau et al. 2012), and $H$ - and $K$-band polarimetric intensities (Canovas et al. 2013; Avenhaus et al. 2014), all led independently to the detection of spiral structures closer to the star, originating at the outer edge of the gap. In Figure 3, we register the contour crests from the spiral of Fukagawa et al. (2006; their Figure 2), as well as the most prominent spiral feature from Casassus et al. (2012; spiral no. 2 in their Figure 2). Our S1 model extends the radio spiral to its NIR root, suggesting that the $H$-band spiral arm, its $K s$-band root, and the inner rim of $\mathrm{S} 1$ are part of the same structure.

\section{DISCUSSION: ORIGIN OF THE SPIRAL ARMS}

Several scenarios have been proposed for the occurrence of spirals in TDs (see, e.g., Boccaletti et al. 2013 for a summary). HD 142527, with its well-defined tightly coiled radio spirals in Keplerian rotation, provides an interesting case study that suggest a different origin than the $\mathrm{CO}$ spirals in the disk of AB Aur.

S1 could be fit with Equations (1) and (2) assuming an embedded companion (Section 2.3.2), although its location on the model spiral could not be precisely constrained. An object has been discovered by Biller et al. (2012) and re-detected in $\mathrm{H} \alpha$ by Close et al. (2014) at 12 AU. Extending S1 inward does not allow us to confirm a possible relationship. Nevertheless, the clearing of a gap as large as $140 \mathrm{AU}$ should involve several planets (e.g., Dodson-Robinson \& Salyk 2011). For S2 and S3, both the relatively poor least-squares fit with Equations (1) and (2) and their very large scales argue in favor of a different cause.

Simulations in the context of protoplanetary disks have shown that two-armed spirals, such as S2 and S3, could be induced by stellar encounters (e.g., Larwood \& Kalas 2001; Quillen et al. 2005). Similarly, simulations of Augereau \& Papaloizou (2004) and Quillen et al. (2005) reproduce the large-scale ( $2525 \mathrm{AU})$ tightly wound spiral observed in the disk of HD 141569 A, assuming tidal induction from one of its M-dwarf companions (external to the disk). Tidally induced spiral structures are transient and vanish within a few orbits, which implies either a very recent encounter or a bound companion external to the disk periodically exciting spirals. For HD 142527 no such object has been detected.

Gravitational instability (G.I.) is an alternative scenario able to create a grand-design two-armed spiral structure (see, e.g., Boss 1998). The stability of a disk is characterized by Toomre's parameter, $Q$ (Toomre 1964). Fukagawa et al. (2013) computed an upper limit of 1-2 for $Q$ in the dust continuum horseshoe, so that depending on the gas-to-dust ratio, fragmentation of either the gas or the dust or both components due to G.I. may occur. Approximating $Q \approx\left(M_{\star} / M_{d}\right) h_{S}$ (Gammie 2001), with the mass of HD $142527 M_{\star}$ set to $2 M_{\odot}$, the mass of the disk $M_{d}$ set to $0.1 M_{\odot}$ (Verhoeff et al. 2011), and the aspect ratio at both spirals $h_{S} \sim 0.1$ (Section 2.3.1), we find $Q \sim 2.0$, similarly to Fukagawa et al. (2013). This suggests that the outer disk in general, not just the horseshoe, is stable but close to the instability regime.

This paper makes use of the following ALMA data: ADS/ JAO.ALMA $\backslash \# 2011$ 1.0.00465.S. ALMA is a partnership of ESO, NSF, NINS, NRC, NSC, and ASIAA. The Joint ALMA observatory is operated by ESO, AUI/NRAO, and NAOJ. Financial support was provided by Millenium Nucleus P10-022-F (Chilean Ministry of Education) and by FONDECYT grant 1130949. The authors acknowledge the referee for his comments.

\section{REFERENCES}

Acke, B., \& van den Ancker, M. E. 2004, A\&A, 426, 151

Augereau, J. C., \& Papaloizou, J. C. B. 2004, A\&A, 414, 1153

Avenhaus, H., Quanz, S. P., Schmid, H. M., et al. 2014, ApJ, 781, 87

Biller, B., Lacour, S., Juhász, A., et al. 2012, ApJL, 753, L38

Boccaletti, A., Pantin, E., Lagrange, A.-M., et al. 2013, A\&A, 560, A20

Boss, A. P. 1998, ApJ, 503, 923

Brinch, C., \& Hogerheijde, M. R. 2010, A\&A, 523, A25

Canovas, H., Ménard, F., Hales, A., et al. 2013, A\&A, 556, A123

Casassus, S., Hales, A., de Gregorio, I., et al. 2013a, A\&A, 553, A64

Casassus, S., Perez, M. S., Jordán, A., et al. 2012, ApJL, 754, L31

Casassus, S., van der Plas, G., Perez, S., et al. 2013b, Natur, 493, 191 Clampin, M., Krist, J. E., Ardila, D. R., et al. 2003, AJ, 126, 385

Close, L. M., Follette, K. B., Males, J. R., et al. 2014, ApJL, 781, L30 Corder, S., Eisner, J., \& Sargent, A. 2005, ApJL, 622, L133

de Gregorio-Monsalvo, I., Ménard, F., Dent, W., et al. 2013, A\&A, 557, A133 de Zeeuw, P. T., Hoogerwerf, R., de Bruijne, J. H. J., et al. 1999, AJ, 117, 354 Dodson-Robinson, S. E., \& Salyk, C. 2011, ApJ, 738, 131

Dullemond, C. P., \& Dominik, C. 2004, A\&A, 421, 1075

Fujiwara, H., Honda, M., Kataza, H., et al. 2006, ApJL, 644, L133

Fukagawa, M., Hayashi, M., Tamura, M., et al. 2004, ApJL, 605, L53

Fukagawa, M., Tamura, M., Itoh, Y., et al. 2006, ApJL, 636, L153

Fukagawa, M., Tsukagoshi, T., Momose, M., et al. 2013, PASJ, 65, L14

Gammie, C. F. 2001, ApJ, 553, 174

Goldreich, P., \& Tremaine, S. 1979, ApJ, 233, 857

Grady, C. A., Muto, T., Hashimoto, J., et al. 2013, ApJ, 762, 48

Grady, C. A., Polomski, E. F., Henning, T., et al. 2001, AJ, 122, 3396

Hersant, F., Wakelam, V., Dutrey, A., et al. 2009, A\&A, 493, L49

Kim, H. 2011, ApJ, 739, 102

Larwood, J. D., \& Kalas, P. G. 2001, MNRAS, 323, 402

Lin, S.-Y., Ohashi, N., Lim, J., et al. 2006, ApJ, 645, 1297

Muto, T., Grady, C. A., Hashimoto, J., et al. 2012, ApJL, 748, L22

Ohashi, N. 2008, Ap\&SS, 313, 101

Perez, S., Casassus, C., Ménard, F., et al. 2014, ApJ, submitted Qi, C., D'Alessio, P., Öberg, K. I., et al. 2011, ApJ, 740, 84 Quillen, A. C., Varnière, P., Minchev, I., et al. 2005, AJ, 129, 2481

Rameau, J., Chauvin, G., Lagrange, A.-M., et al. 2012, A\&A, 546, A24

Rosotti, G. P., Ercolano, B., Owen, J. E., et al. 2013, MNRAS, 430, 1392

Tang, Y.-W., Guilloteau, S., Piétu, V., et al. 2012, A\&A, 547, A84

Toomre, A. 1964, ApJ, 139, 1217

Verhoeff, A. P., Min, M., Pantin, E., et al. 2011, A\&A, 528, A91

Zhu, Z., Nelson, R. P., Dong, R., et al. 2012, ApJ, 755, 6

Zhu, Z., Stone, J. M., \& Rafikov, R. R. 2013, ApJ, 768, 143 